\title{
"Loüer celluy qui demeure là-haut": la forme de l'hymne ronsardien
}

\author{
JEAN CÉARD
}

Au début de l" "Hymne de Calaîs et de Zetes", Ronsard déclare tout net son intention de consacrer cette pièce aux deux fameux "enfans de Borée". Puis, se reprenant, il reconnaît qu'au lieu de se contenter de dédier cet Hymne à Marguerite de France, il devrait plutôt chanter son mérite,

Sans loüer autre nom, et des Grecs mensongers,

N'emprunter desormais des sujets estrangers

d'autant qu'un tel éloge suffirait à assurer la gloire de qui l'entreprendrait. Mais, continue-t-il,

vous le dedaignez, et dites qu'il ne faut Sinon loüer celluy qui demeure là-haut.

De qui la gloire doit tousjours estre chantée.

Cette dédicace se poursuit par un long passage où, après avoir fait observer à la princesse que, si elle refuse d'être louée, il lui faut, en somme, n'être pas louable, le poète proteste que, pour sa part, et qu'elle le veuille ou non, il ne renoncera pas à dire sa louange! Après quoi, commence, presque abruptement, l'Hymne des deux fils de Borée.

Étrange début, auquel il peut sembler paradoxal d'emprunter un titre puisque l'Hymne qui suit paraît aussi étranger aux voeux de la Princesse qu'aux protestations du poète. Et pourtant cette périphrase - "celluy qui demeure là-haut" - qui ne nomme pas Dieu et se contente de le désigner par l'éloignement de sa haute demeure, ${ }^{1}$ est peut-être révélatrice de l'entreprise des Hymnes de Ronsard: comment chanter l'Inaccessible, comment nommer l'Innommable?

Quand et en quels termes Ronsard conçut-il le projet des Hymnes? Il est difficile de le dire, mais du moins peut-on rappeler le fait, souvent noté, qu'une des pièces du recueil de 1555,1 ' "Hercule Chrestien", était en projet 


\section{2 / Renaissance and Reformation}

en juin 1553. On le sait par une lettre du médecin Pierre des Mireurs qui, justifiant Ronsard de la récente publication des Folastries, ajoute: "J'ai pleine confiance de voir quelque jour notre Terpandre, dans son humanité, chanter d'un vers mieux venu la fameuse geste de l'Hercule Chrétien". ${ }^{2}$

Il est très probable qu'à cette date Ronsard est sensible aux observations de tout un courant idéologique qui, en poésie, combat le lyrisme mythologique tel que Ronsard l'a jusque-là conçu, et veut promouvoir une véritable poésie religieuse. Dans ces débats, deux voix méritent une particulière attention, celle de Nicolas Denisot et celle de Jodelle, puisque le premier est l'auteur d'un sonnet à Ronsard "sur son Hercule Chrestien" qui, jusqu'en 1584, ne cessera de précéder immédiatement l'Hymne du poète, et que le second coinpose, pour le recueil des Hymnes de 1556, une longue épitre liminaire que Ronsard maintiendra constamment, la disposant à partir de 1560 en tête de la section des Hymnes. Les idées de ces deux auteurs ayant été parfaitement étudiées, ${ }^{3}$ quelques rappels suffiront. Denisot, auteur de Noëls (1545) et, en 1553 précisément, de Cantiques du premier advenement de Jésuschrist - relevons en particulier ce titre: "Chant triomphal montrant qu'en la venue de Jésuschrist tous les idoles anciens tombèrent" - condamne une poésie qui s'attarde à parler païen: les dieux des Grecs et des Romains sont morts; sans doute même n'étaient-ils que des démons. Si l'on peut encore s'inspirer de la mythologie, c'est seulement dans la mesure où les fables païennes semblent, sous leurs oripeaux, cacher quelques pressentiments du christianisme; mais il faut que la lyre chrétienne les y décèle et les replace dans leur vraie lumière. Quant à Jodelle, qui, du reste, a fortement subi l'influence de Denisot, il publie, dans les Cantiques de celui-ci, une Ode importante qui soutient, pour l'essentiel, les mêmes thèses: ${ }^{4}$ si une sainte fureur a animé Orphée, Amphion et Arion, nous savons, depuis l'avènement du Christ, que c'est Dieu lui-même qui est descendu aux Enfers et, "sous nostre forme", a vaincu la mort. Que nous servent désormais les fables antiques? "Fuyons ces vois menteresses", et écoutons Denisot, "Prince des hymnes sainctes".

Ces thèses - certes plus complexes que ce bref résumé ne le laisse supposer - se retrouvent assurément dans l' "Hercule Chrestien". Les premiers vers suffisent à prouver que Ronsard a entendu ces pressants appels et en mesure l'importance:

Est-il pas temps desormais de chanter

Un vers chrestien qui puisse contenter,

Mieux que devant, les chrestiennes oreilles?

Il approuve aussi l'idée que le lyrisme à l'antique est périmé: 
Le payen sonne une chanson payenne,

Et le chrestien une chanson chrestienne:

Le vers payen est digne des payens,

Mais le chrestien est digne des chrestiens. ${ }^{5}$

Toute son entreprise, exploitant peut-être une suggestion de Denisot, ${ }^{6} \mathrm{va}$, pour ce qui est d'Hercule, consister à montrer, à travers le récit de sa vie et de ses exploits, quelles déformations les Païens idolâtres ont fait subir aux révélations qu'inspirées par Dieu leur ont dispensées les Sibylles. Que cette thèse soit loin d'être le simple écho des théories de Denisot ou de Jodelle, on le voit facilement. ${ }^{7}$ Il est certain que Ronsard ne s'est pas contenté de satisfaire à une requête contemporaine et que son Hymne tout entier suppose une méditation personnelle, solidement informée, où a dû s'élaborer le projet des Hymnes. Il propose, en effet, une sorte d'interprétation typologique, si l'on peut dire, à rebours: il ne s'agit plus ici de lire dans les fables des pressentiments de la vérité, pressentiments qui répondraient à une révélation progressive de Dieu nous donnant d'abord à lire comme l'ombre de la vérité; mais il ne s'agit pas non plus de déceler l'action des diables qui, connaissant à l'avance la vérité, en auraient, dans le paganisme, inscrit l'écho déformé, pour maintenir les hommes dans l'erreur et leur inspirer une sorte de nonchalance du salut. ${ }^{8}$ Ronsard, lui, accuse l'ingratitude des hommes, et aussi bien une sorte d'incapacité à concevoir la vérité sans la dévoyer.

Ces deux griefs méritent quelques précisions: ingrats, certes, les Juifs, qui, plus que les Gentils, auraient dû croire les Prophètes, et pourtant les ont fait massacrer. C'est quand Dieu les a vus "retifz / A leur salut" qu'il a envoyé aux Gentils les Sibylles pour leur faire prophétiser l'avènement du Christ:

Mais, ô Seigneur, les Gentilz vicieux

Qui n'avoient point ta foy devant les yeux

Ont converty les paroles predittes

(Que pour toy seul la Sybille avoit dittes)

A leurs faux Dieux, contre toute raison.

Maladresse de Dieu, qui devait penser que les Gentils étaient encore plus incapables que les Juifs de recevoir son message? Ronsard trouverait sans doute la question impertinente. Son Dieu ne se tourne vers les Gentils que parce que les Juifs ont déçu son attente: car c'est un Dieu avide de se faire connaître aux hommes, reconnaître des hommes; s'il a fait "pour nous" le monde, les étoiles et les deux luminaires, s'il a "pour nous" ordonné la course de l'année, c'est, dit Ronsard s'adressant au Seigneur, tout autant pour notre usage que 


\section{4 / Renaissance and Reformation}

Pour nous monstrer par son train regulier, Combien tu es en tes faictz singulier.

De même, si Dieu a voulu prendre le fardeau de notre humanité, lui dit-il encore,

avant que le prendre,

Tu nous le feis par tes heraux entendre.

Dans l'incarnation du Christ, Ronsard souligne tout autant notre rédemption qu'une manifestation merveilleusement prochaine de Dieu, comme s'il fallait réduire la distance infinie qui sépare Dieu et les hommes et met ceux-ci dans l'incapacité d'entendre la plénitude de la vérité.

D'une certaine manière, il faut vaincre un double obstacle: celui du mensonge dont les hommes enveloppent les messages prophétiques - et donc montrer

Que la plus-part des choses qu'on escrit

D'Hercule, est deue à un seul JESUSCHRIST - ;

et l'obstacle qui vient de cette sorte d'immensité de Dieu en raison de laquelle nous détaillons ses bienfaits en autant de noms, par inaptitude à le nommer tout à fait:

Hercule fut en châcune contrée,

Où, par effect, sa force il a monstrée,

Tousjours nommé des hommes, en faveur

De ses vertus, chasse-mal, et sauveur:

De mesmes noms JESUSCHRIST on surnomme,

Car seul il garde, et seul il sauve l'homme.

Il n'est pas inadéquat d'utiliser ces noms de chasse-mal et de sauveur; l'erreur, c'est de les rapporter à Hercule, et non à Jésus-Christ. Cette réflexion sur les noms divins s'inscrit dans une longue tradition; d'autres textes, comme l' "Hymne de la Philosophie", s'inspirant du De mundo du pseudo-Aristote, il est probable que Ronsard y a noté ce thème, cher à la pensée hellénistique, d'un Dieu unique, mais qui porte une multitude de noms en raison de la diversité de ses effets: "Dieu est unique, dit le $D e$ mundo, 401 a 12, mais il porte une multitude de noms car il en reçoit autant qu'il y a d'effets ( $\pi \bar{\alpha} \theta \varepsilon \sigma i$ ) nouveaux dont il se montre la cause." Il avait, du reste, lu sans doute une glose de Servius (ad Georg. I,5): "Les Stoïciens disent qu'il n'y a qu'un seul Dieu, et une seule et même puissance (divine), qui reçoit des noms différents en conséquence de ses fonctions (pro ratione officiorum). De là vient que c'est le même être qu'on appelle Soleil, Apollon, 
Bacchus, le même qu'on appelle Diane, Cérès, Junon, Proserpine". 9 C'est de ces textes - nombreux - que Ronsard se fait directement l'écho dans l'Abbregé de l'Art Poëtique François (Lm, XIV, 6): "Car les Muses, Apollon, Mercure, Pallas et autres telles deitez ne nous representent autre chose que les puissances de Dieu, auquel les premiers hommes avoyent donné plusieurs noms pour les divers effectz de son incomprehensible majesté." Mais, dès 1555, l' "Hymne de la Justice" disait déjà (Lm, VIII, p. 69):

Car Jupiter, Pallas, Apollon, sont les noms

Que le seul DIEU reçoit en meintes nations

Pour ses divers effectz que l'on ne peut comprendre,

Si par mille surnoms on ne les fait entendre.

Mais, si Dieu est ainsi "polyonyme" ou même "panonyme", c'est qu'il est, au fond, "anonyme", remarquent divers passages du Corpus Hermeticum: "Il est, lui, le Dieu trop grand pour avoir un nom, il est l'inapparent et le très apparent; lui que contemple l'intellect, il est aussi celui que voient les yeux; il est l'être sans corps, l'être aux corps multiples, ou plutôt l'être qui a tous les corps. Rien n'existe qu'il ne soit aussi: car tout ce qui est, tout est lui. Et de là vient qu'il a tous les noms parce que toutes choses sont issues de cet unique père; et de là vient qu'il n'a point de nom parce qu'il est le père de toutes choses". 10

"Il est l'inapparent et le très apparent; lui que contemple l'intellect, il est aussi celui que voient les yeux": c'est assez bien définir l'appréhension de Dieu qui s'exprime dans les Hymnes. Ronsard n'a jamais renouvelé l'essai de l' "Hercule Chrestien", sans pourtant jamais récuser cette pièce. L' "Hercule Chrestien" est utile en ce qu'il dit la nécessité d'une poésie chrétienne; mais, s'il désigne bien le but, il ne montre pas la voie. Du reste, tout en le maintenant, Ronsard l'a disposé à la fin du livre de 1555: à cette place, il illustre ce moment unique où l'humain et le divin se rencontrent. Mais tout le reste du recueil, qui semble emprunter les cadres principaux de sa pensée à des textes comme le De mundo, médite sur cet étrange mélange d'éloignement et de proximité qui caractérise la relation de l'humain et du divin, sur cette sorte d'inaccessibilité d'un Dieu qui ne se donne à connaître que médiatement à un homme avide d'instaurer avec lui une relation plus immédiate, sur ce Dieu qui est à la fois "l'inapparent et le très apparent".

Qu'il soit très apparent, c'est ce qu'illustre abondamment tout un pan des Hymnes. Ce n'est pas seulement l' "Hercule Chrestien" qui nous engage à contempler le bâtiment ordonné du monde, et à y lire à la fois l'évidence d'un Dieu qui l'a ordonné et la providence d'un Dieu qui, pour nous, l'a 
ordonné. Irrité de l'ingratitude des hommes, Dieu, dans l' "Hymne de la Justice" (p. 61), rappelle que l'homme connaît ses bienfaits, sait que toui a été fait "pour luy seul":

J'ay fait pour luy du Ciel le grand Tour nompareil,

Les Estoilles, le Jour, la Lune, et le Soleil

Pour luy donner clarté.

De lui aussi, comme le rappelle, à la fin de la même pièce (p.68), le cardinal de Lorraine, viennent les "règles" et les "lois" auxquelles se soumettent tous les animaux; de lui encore, l'ordre que garde chaque élément et qui l'entretient dans son être par "un tresfidelle accord". De lui, ou plutôt de la Nature, car, précise Ronsard,

Nature venerable en qui prudence abonde

A fait telle ordonnance en l'Ame de ce Monde,

Qui ne se change point, et ne se changera,

Tant que le Ciel vouté la Terre logera.

Avant qu'il ne naisse un nouveau ciel et une nouvelle terre, subsistera cet ordre immuable. Ici encore on perçoit un écho des spéculations héllénistiques auxquelles la Renaissance porte tant d'intérêt: "Si Dieu, dit le $D e$ mundo, 397 b 21 sqq., est bien réellement le conservateur et le créateur de tout ce qui, de quelque façon que ce soit, est accompli dans ce monde visible, ce n'est pas pourtant qu'il endure la fatigue d'un travailleur manuel et d'un homme de peine: non, il a à son service une force que rien n'use, grâce à laquelle il étend son pouvoir jusqu'aux objets qui semblent loin de lui." Cette force, c'est ici la Nature, elle-même ordonnatrice de cette autre force déléguée qu'est l'Âme du Monde. Soumise à Dieu, elle le dispense de peiner à la façon "d'un homme de peine". Telle est exactement l'image que, de la Création, nous propose l' "Hymne de la Mort" (p. 165):

Mais le Soleil, la Lune et les Astres des Cieux

Font avecque travail leur tour laborieux:

La Mer avec travail deux fois le jour chemine,

La Terre, tout ainsi qu'une femme en gesine

Qui avecques douleur met au jour ses enfans,

Ses fruictz avec travail nous produit tous les ans:

Ainsi Dieu l'a voulu, à fin que seul il vive

Affranchy du labeur.

Au labeur ordonné mais douloureux de la Création tout entière s'oppose cette sorte d'oisiveté de Dieu qui commet à ses délégués le soin d'étendre sur toute chose son pouvoir.

Ces délégués, les Hymnes s'occupent à les recenser. C'est, par exemple, le 
Ciel, dont la forme ronde même, signe de perfection, marque qu'il est directement animé et mis en mouvement par "l'Esprit de l'Eternel" luimême (p. 142); qu'il accomplisse une fonction d'origine divine, c'est ce qu'atteste son aptitude à harmoniser les contraires (p. 143):

\footnotetext{
De ton bransle premier, des autres tout divers,

Tu tires au rebours les corps de l'Univers,

Bien qu'ilz resistent fort à ta grand' violence,

Seulz à-part demenans une seconde dance,

L'un deçà, l'autre là, comme ilz sont agitez

Des discordans accordz de leurs diversitez.
}

Ronsard ne manque pas, ensuite, de recourir au thème de l'harmonie musicale. Le De mundo 396 a-b, lui aussi, orchestre largement l'idée de la concordia discors, sans omettre l'harmonie: "On s'est demandé avec surprise comment il peut jamais se faire que le monde, alors qu'il est constitué de principes contraires $(\ldots)$, n'ait pas été depuis longtemps détruit et réduit à néant $(. .$.$) ;$ Peut-être la nature a-t-elle du penchant pour les contraires, et est-ce de cela même qu'elle tire l'harmonie, et non pas des semblables (... ). C'est en fondant ensemble les sons aigus aux graves, les longs aux brefs, que la musique, avec des voix diverses, produit une harmonie unique."

Dans l'ordre humain, semblablement, l'ordre divin se reflète. C'est le sens même de la figure du roi, que Ronsard dresse à la tête du recueil de 1555. Non seulement le roi réunit en lui toutes les vertus dispersées entre les grands qui constituent son entourage (p. 8), non seulement il est lui-même unifié, rassemblant en sa personne les biens du corps et ceux de l'esprit, mais encore il est celui qui, malgré les menées de l'injustice et de l'ambition, à l'intérieur comme à l'extérieur, maintient l'ordre; la composition de cet Hymne, qui campe d'abord la haute image du roi, le présente comme étant d'abord, en lui-même, une figure d'ordre, et c'est cet ordre incarné en sa personne qui semble comme rayonner de lui, se diffuser autour de lui, instaurant l'harmonie.

Levant plus haut encore le regard, Ronsard disposera, au seuil des Hymnes de 1556, la figure de l'Éternité. L' "Hymne du Ciel" y préparait en donnant à voir dans "l'Esprit de l'Eternel" la source de l'animation et du mouvement du Ciel. L' "Hymne de l'Éternité" (p. 247) lui donne le Ciel en partage. Que Ronsard désigne par "l'Esprit de l'Eternel" le premier Moteur d'Aristote ou l'Âme du Monde des néoplatoniciens, "1 il veut surtout saisir, au-delà du Ciel, dont les révolutions engendrent le temps, non pas tout à fait Dieu, mais le mystère de son être, l'interminabilité et la simultanéité conjointes, puisque l'Éternité est

celle qui jamais pour les ans ne se change,

Mais bien qui faict changer les siecles et les temps. 


\section{8 / Renaissance and Reformation}

De toutes les figures de Dieu, celle-ci est sans doute la moins inadéquate. L'Éternité, en effet, est conservatrice du monde, qu'elle anime, lui donnant vie et force, et qu'elle harmonise "en eternelle paix" (p.251). Mais, en même temps, elle connaît l'oisiveté de Dieu (p. 247):

Sans rien faire tu vis en tous biens plantureuse.

Le regard ne peut pas se porter plus haut. Certes, dit l' "Hymne du Ciel" (p. 146), Dieu n'a pas bâti sa maison hors de la clôture du monde, mais s'y est logé, - comme l'Éternité, quant à elle, est assise (p. 248)

Tout au plus haut du Ciel dans un throsne doré,

ce qui, à nouveau, fait songer au Demundo, 400 a 5 sq.: Dieu, dit ce traité, "se trouve dans un lieu pur, que nous appelons, au vrai sens du mot, Ciel ( obe $\alpha$ vōv ) du fait qu'il est la frontière supérieure ( ó́pov tòv 'ó $v \omega$ ) du monde". Mais, de ce Dieu, nous savons, parle monde, l'existence; l'essence nous échappe. Comme dit Philon, nous "appréhendons Dieu par son ombre". ${ }^{12}$ De l'Éternité elle-même, nous ne pouvons guère approcher que par la voie négative (p. 254):

Tu es toute dans toy, ta partie, et ton tout,

Sans nul commencement, sans meillieu, ne sans bout,

Invincible, immuable, entiere, et toute ronde,

N'ayant partie en toy, qui dans toy ne responde,

Toute commencement, toute fin, tout meillieu,

Sans tenir aucun lieu, de toute chose lieu.

Notons au passage qu'une fois encore le De mundo, $401 \mathrm{~b}$, avait choisi pour terminer de citer ce passage particulièrement célèbre des Lois de Platon (IV, 715 e): "La Divinité qui, suivant l'antique tradition, tient en mains le commencement, la fin et le milieu de tout ce qui existe"... Apulée traduisait: "Deus (...) principia et fines et media rerum omnium penetrat" ${ }^{13}$ En substituant au penetrat un est, Ronsard tente de s'approcher de l'essence divine, mais dès lors il lui faut tenir également et simultanément la proposition contraire: "Sans nul commencement, sans meillieu, ne sans bout". Car la lumière de Dieu sort de son obscurité. Le très apparent est aussi l'inapparent.

"Lui que contemple l'intellect, il est aussi celui que voient les yeux", disait le De mundo. Ronsard n'a pas négligé cette double voie. On peut même dire que la voie de la contemplation de l'intellect qu'emprunte l' "Hymne de la Philosophie" s'exprime en des termes qui sont particulièrement proches du De mundo en son début, comme le savant Richelet s'en était justement avisé. Ronsard écrit (pp. 86-87): 
Elle, voyant qu'à l'homme estoit nyé

D'aller au Ciel, disposte, a delié,

Loing, hors du corps, nostre Ame emprisonnée,

Et par esprit aux astres l'a menée,

Car en dressant de nostre Ame les yeux,

Haute, s'attache aux merveilles des Cieux,

Vaguant par tout, et sans estre lassée

Tout l'Univers discourt en sa pensée.

Et le De mundo: “Puisqu'il n'était pas possible avec le corps d'atteindre jusqu'aux lieux célestes ni de laisser derrière soi la terre (...), l'âme, elle du moins, par la philosophie, quand elle eut pris pour guide l'intellect, a traversé l'immensité, elle a accompli le voyage, car elle a découvert un chemin sans fatigue (...); par son regard intérieur, qui est divin, elle a appréhendé les choses divines." Notons cependant une différence: à cette appréhension aisée du divin, Ronsard répond seulement par l'idée que la Philosophie, au plus haut de son vol, s'allie aux astres (p. 87),

Osant de DIEU la nature espïer.

Aurait-il lu Philon, qui, célébrant, lui aussi, la montée victorieuse de l'intellect à travers le cosmos, souligne qu' "il désire d'aller toujours plus outre, et d'appréhender, s'il se peut, l'essence de Dieu, dont on ne peut rien percevoir, sinon le fait qu'il existe"? ${ }^{14}$

Quoi qu'il en soit, Dieu apparaît bien, selon le sens que nous avons proposé de donner à cette périphrase, comme "celluy qui demeure là-haut". De même qu'il ne se donne à connaître que médiatement, de même on ne peut le louer que médiatement. Toute louange de Dieu ne peut être qu'oblique, indirecte.

En outre, de ce Dieu, lointain puisque son essence nous échappe, mais proche aussi puisque nous l'appréhendons du moins par son ombre, est-il possible d'éprouver, au plus près de nous, la providence? On sait que la lecture du De mundo a poussé les Pères de l'Église à accuser Aristote d'avoir borné l'action de la Providence en lui soustrayant la région sublunaire. ${ }^{15}$ Cette "accusation" ne pourrait-elle atteindre aussi Ronsard?

Mais il faut tout de suite ajouter que Ronsard ne s'y résigne pas, et que de là vient cette sorte de ferveur inquiète, ou d'inquiétude fervente, qui anime les Hymnes. Sans nul doute se représente-t-il le monde comme séparé en deux régions qui ont des traits vivement contrastés: à l'une, l'incorruptibilité, et, pour ceux qui y demeurent, le "contentement"; 16 à l'autre, le changement et la corruption, et, pour ses habitants, la mort et le malheur. J'ai montré ailleurs que la disposition des livres des Hymnes procédait de cette opposition..$^{17}$ Il suffira de marquer ici que cette opposition n'est pas 
posée, dans les Hymnes, comme une idée simplement capable d'ordonner le discours, mais plutôt comme, en soi-même, une énigme - insupportable: comment admettre que ce Dieu qui a tout fait pour nous nous ait comme assignés à résidence dans la partie la plus misérable de la création?

S'il convient certes de reconnaître que nulle part Ronsard ne dit positivement que la région sublunaire est soustraite à la providence divine, du moins doit-on aussitôt ajouter que les signes qui attestent clairement de sa présence agissante nous font défaut. La "Prière à la Fortune" consiste, en somme, à promettre à cette déesse de ne l'honorer comme la Providence que si, consentant aux voeux du poète, elle cesse de se conduire comme elle l'a toujours fait! Polyonyme, elle aussi (v. 84-85, p. 106), "commencement et bout / De toute chose" (v. 90-91, p. 106), elle est comme une image ironique de la Divinité puisqu'à ces traits elle ajoute le manque de "foy", une boule sous les pieds, un bandeau sur les yeux, des ailes, autant de marques de l'inconstance et du caprice. Que dire, de même, de la Mort? Tout l' "Hymne de la Mort" dit clairement qu'il est trop facile de la louer simplement comme ce qui met un terme à notre exil, et que, s'il faut la célébrer comme un bien, ce n'est qu'au prix d'un presque impossible oubli de l'attachement que nous portons à la vie. Veut-on encore une autre preuve de l'étrange comportement de la Providence? Dieu, assure l' "Hymne des Astres", n'a pas omis de multiplier, à l'endroit de chacun de nous, les signes de sa providence particulière, nous faisant connaître ses volontés, non seulement par les Astres,

Mais par l'onde et par l'air et par le feu trespront,

Voire (qui le croira) par les lignes qui sont

Escrites dans noz mains, et sur nostre visage (p. 159);

malheureusement, ces signes nous sont illisibles:

Mais faute de pouvoir telles lignes entendre,

Qui sont propres à nous, nous ne povons comprendre

Ce que Dieu nous escrit, et sans jamais prevoir

Nostre malheur futur, tousjours nous laissons choir

Apres une misere, en une autre misere.

Curieuse Providence qui inscrit si soigneusement en nous-mêmes les signes détaillés de "noz mauvais et noz bons accidens", mais néglige de nous en permettre le déchiffrement!

On n'est guère étonnné que, dans ces conditions, la montée de l'âme à travers le cosmos, que décrit l' "Hymne de la Philosophie", n'ait pas tout à fait même tonalité que dans les textes anciens qui développent à l'envi ce lieu commun. Quand, pour vanter cette puissance de l'intellect, Ronsard dit que la Philosophie (p. 99) 
tout le Ciel fait devaller en terre,

Et sa grandeur en une sphere enserre

(Miracle grand) qui tant d'astres contrains,

Comme un joüet, nous met entre les mains,

il se pourrait qu'il emprunte cette "conception" à l'Icaroménippe de Lucien; mais faut-il rappeler que ce texte - bien connu à la Renaissance (Érasme l'a traduit) - est une parodie des voyages de l'âme à travers le cosmos? Et que dire de la manière dont Ronsard présente cette victoire?

Et comme on voit la sorciere importune

Tirer du Ciel par ses charmes la Lune,

Elle, sans plus, la Lune ou le Soleil

N'attire à bas par son art nompareil,

Mais tout le Ciel fait devaller en terre, etc.

Surprenante comparaison qui, pour éclairer l'idée de la puissance de la Philosophie, ne trouve rien de mieux que de la dire supérieure à l'art de la "sorciere importune". ${ }^{18} \mathrm{Au}$ demeurant, la suite ajoute que la Philosophie défie Jupiter et se rend maîtresse de la "cheine ferrée" à laquelle celui-ci tient le monde suspendu. Voilà ce que devient un lieu commun qui, dans de nombreux textes anciens, ${ }^{19}$ a pour but de prouver que, si l'intellect a un tel pouvoir, a fortiori la pensée de Dieu peut partout s'étendre, - et permet donc de conclure sans restriction à la Providence.

Deux pièces des Hymnes de 1555 méritent ici une mention: 1' "Hymne des Astres" et "les Daimons". Parmi les délégués de Dieu, que recense Ronsard, Astres et Daimons ont des fonctions spécifiques: l'attention que Dieu porte aux hommes l'a conduit à confier aux premiers "le fil des Destinées" (p. 154), et aux seconds - ou, du moins, à une partie d'entre eux - une explicite fonction de médiation (p. 126). Pourtant, c'est peu de dire que les Astres exercent sur nous une autorité contraignante, accablante, - et Ronsard énumère longuement (pp. 155-158) les divers états qu'ils nous imposent, comme si, content d'avoir délégué son pouvoir, Dieu se désintéressait de nous. Quant aux Daimons, faut-il rappeler que, d'abord cantonnés dans la région de l'air, ils se mettent à proliférer, avides de métamorphoses, curieux de malignes ou méchantes espiègleries? Ces êtres, dont le début de la pièce disait qu'ils étaient, dans l'ordre du monde, les habitants nécessaires de l'air, il faudra, pour finir, prier le Seigneur de nous en délivrer, en les invitant à aller ailleurs porter leurs obsédantes assiduités.

D'un mot, on dirait qu'à mesure que l'Un se diffuse, au lieu d'ordonner le Multiple, il s'éparpille, se disperse en lui, comme si l'ordre, au lieu de succéder à une simple absence d'ordre, avait à lutter contre une sorte de désordre actif. Désordre sans nom, "Discord", comme dit souvent Ronsard, ${ }^{20}$ qui, à l'ordre, oppose comme une sourde résistance. C'est de lui que, 
dans l' "Hymne de Henry II", doit triompher le roi; c'est lui qui, dans l' "Hymne de la Justice", est responsable de l'exil de Justice. Du reste, tout le premier livre des Hymnes pourrait être décrit comme le théâtre, aux scènes diverses, de cette sourde lutte, et on est tenté de déceler ici quelque pente vers un dualisme qui n'est pas sans faire penser à certaines gnoses. Si l'hypothèse est juste, le second livre s'éclaire: il fait abruptement succéderà l' "Hymne de l'Éternité", dont il a déjà été question, ce qu'il est convenu d'appeler deux hymnes mythologiques; ceux-ci, qui sont explicitement liés (p. 292), racontent les aventures de Calaïs et de Zéthès, de Pollux et de Castor, tous Argonautes; il se peut, comme on l'a suggéré, ${ }^{21}$ qu'ils peignent, en fait, les aventures de l'héroïsme et la tentation d'indépendance aveugle, d'individualisme trouble, qui l'habite. En ce cas, l'écart est immense entre l'Éternité et son cortège ordonné, d'une part, et, d'autre part, ces héros gagnés par l'esprit de rivalité et qui apparaissent si différents de ceux dont le premier livre édifiait le Temple. Et, comme pour mieux marquer cet écart, Ronsard associe l'éloge de Marguerite de France à la célébration de l'Éternité (p. 247), alors qu'il donne le premier des deux hymnes mythologiques comme le simple substitut de l'éloge que la Princesse refuse, mais tout aussi bien de la seule louange qu'elle recommande, celle de "celluy qui demeure là-haut".

Puisqu'on ne peut pas nommer l'Innommable, il reste à l'épier, et à l'épier du lieu de cette terre basse où nous sommes. Telle semble être la réponse de Ronsard à la requête d'une grande poésie religieuse qui se fait entendre de son temps. À la demande d'une poésie religieuse, je dirais volontiers qu'il a répliqué par une poésie théologique. Encore faut-il s'entendre sur ce terme. La critique a multiplié les expressions pour désigner la poésie des Hymnes: poésie cosmologique, poésie scientifique, poésie didactique, poésie philosophique, etc. Toutes ces formules sont contestables. En proposant celle de poésie théologique, je fais référence à toute une tradition antique qui appelle théologie le discours sur les dieux, soit qu'il s'exprime dans les fables des poètes, soit que, discours des savants, il traite des choses divines; ces deux sens distincts mais conjoints sont bien connus à la Renaissance: le premier est utilisé par Ronsard lui-même quand, dans l'Abbregé de l'Art Poëtique (Lm, $\mathrm{XIV}, 4)$, il note que "la Poësie n'estoit au premier aage qu'une Theologie allegoricque"; le second est illustré par Dorat (bien qu'écrivant en vers il évite ce terme technique), quand, dans un liminaire des Hymnes, il note qu'après l'inspiration lyrique et l'inspiration héroïque, Ronsard maintenant chante "les doctes cantiques de la nature" et qu'il l'invite à dire les "coelestia" (pp.4-5). Le De mundo appelle justement théologie le discours sur le monde et les choses célestes. D'une certaine façon, le poète des Hymnes ranime ainsi la tradition du poeta theologus. 
C'est sans doute une version originale de la poésie religieuse que recommandait un Denisot, même si ce n'est peut-être pas tout à fait celle qui était attendue de lui. Ajoutons qu'il n'y a pas lieu de le regretter. Guy Demerson remarque "que c'est dans le désarroi d'un grave accès de maladie que nombre de poètes, d'abord séduits par l'éclat du style paganisant, se sont mis à renier solennellement les faux dieux qu'ils avaient invoqués": 22 aux poètes qu'il cite on pourrait joindre Ronsard, auteur d'une pièce que nous a conservée Pontus de Tyard sous le titre de "Prière à Dieu faicte par Monsieur de Ronsard estant malade"; ${ }^{23}$ Monsieur de Ronsard ne manque pas d'y récuser

Mille Dieux abuseurs que peint la Poësie.

Mais, avouons-le, cette pièce ne manque pas à son génie.

Université de Paris XII

Notes

N.B. Toutes les références aux Hymnes de Ronsard renvoient à l'édition Laumonier, S.T.F.M., t. VIII. On se contente, dans le texte, d'indiquer, entre parenthèses, la page intéressée de cette édition.

1 Ce n'est qu'à partir de 1578 que ce vers devient "Loüer le Dieu qui demeure là-haut". Indice d'une évolution de Ronsard? L'édition de 1587 comportera, outre l' "Hymne des Peres de Famille, à Monsieur S. Blaise" et l' "Hymne de Monsieur Sainct Roch" (Lm, XVIII, 275 et 280), une pièce-préface (Lm, XVIII, 263), souvent citée, qui exprime le voeu quà la façon des Grecs, les Chrétiens chantent

La memoire et les faicts de nos Saincts immortels.

Selon Binet, Ronsard, à la fin de sa vie, “avoit envie, si la santé et la Parque l'eussent permis, d'escrire plusieurs œuvres Chrestiennes" (Vie de Ronsard, éd. Laumonier, pp. 47. 48: texte de 1587 et 1597$)$.

2 "Plane confido (quae est Terpandri nostri humanitas) hunc aliquando Christiani Herculis res praeclare gestas feliciore versu decantaturum." Cette lettre, datée du 30 juin 1553, à Jean de Morel a été publiée par P. de Nolhac, RHLF VI (1899), p. 358. Voir aussi P. de Nolhac, Ronsard et l'Humanisme (Paris: Champion, 1921), pp. 189-191; Laumonier, Ronsard, Poère lyrique, $4^{*}$ éd. (Paris: Hachette, 1932), p. 165; Guy Demerson, La Mythologie dans l'œeuvre lyrique de la Pléiade (Genève: Droz, 1972), p. 398.

3 Voir notamment G.Demerson, op. cit., pp. 257-266.

4 E. Jodelle, O.C., édition Balmas (Paris: Gallimard, 1965) t.I, pp. 78-83.

5 Voir Cl. Faisant, "Le sens religieux de l'Hercule Chrestien", in Autour des Hymnes de Ronsard (Paris: Champion, 1984), p. 245.

6 Voir Cl. Jugé, Nicolas Denisot du Mans, thèse, Caen, 1907, (Le Mans: A. Bienaimé-Leguicheux; Paris: Lemerre, 1907), p. 84; G. Demerson, op. cit., p. 261.

7 Voir Cl. Faisant, art. cit.

8 C'est l'hypothèse de Claude Faisant, art. cité, pp. 255-256, qui reconnaît toutefois que "Ronsard ne fait assurément aucune allusion à cette théorie".

9 Voir aussi Servius, ad Aen. IV, 638. Sur toute cette question, voir AJ. Festugière, La Révélation d'Hermès Trismégiste (Paris: Les Belles-Lettres, 1981), pp. 515-518. Ces textes ont divers échos à la 


\section{4 / Renaissance and Reformation}

Renaissance: Coelius Rhodiginus, Ant. Lect., XXII, 3; H.C. Agrippa, De occ. phil., III, 10; etc.

10 C. H., V, 10; voir aussi Asclepius, 20.

11 Voir F. Joukovsky, "Temps et Éternité dans les Hymnes", in Autour des Hymnes de Ronsard, op. cit., p. 57.

12 Philon, L. A., III, 99.

13 Apulée, De mundo, 374.

14 Philon, Det., 89.

15 Voir A. J. Festugière, L'Idéal religieux des Grecs et l'Évangile (Paris: J. Gabalda, 1932) pp. 224 sqq.

17 Voir J. Céard, "La disposition des livres des Hymnes de Ronsard", in Les Hymnes de Ronsard, Textuel 34/44 no 1 (1985), pp. 83-99.

18 Dans un contexte certes différent, Plutarque, De def. orac., $416 \mathrm{~F}$, une même comparaison: ceux qui nient l'existence des démons détruisent l'ordre du monde "en introduisant la divinité dans les vicissitudes et les affaires humaines et en la faisant descendre pour répondre à nos besoins, comme les Thessaliennes, dit-on, font descendre la lune“.

19 Ainsi Xénophon, Mem., I, 4, 17.

20 Ainsi Laumonier, VIII, p. 249 (v. 60).

21 D. Ménager, "Ordre et variété dans les Hymnes mythologiques", in Les Hymnes de Ronsard, op. cit., (voir n. 17), pp. 101-112.

22 Guy Demerson, op. cit., pp. 266-267.

23 Paul Laumonier ne l'a pas recueillie dans l'édition S.T.F.M.des O.C. de Ronsard, mais il l'avait transcrite dans l'édition Lemerre, t. VI, pp. 506-507. Une mention dans Nolhac, Ronsard et l'humanisme, op. cit., p. 200, n. 3. 